\title{
PANEL DISCUSSION ON THE CLASSIFICATION AND TERMINOLOGY OF STELLAR POPULATIONS
}

\author{
IVAN R. KING (ORGANIZER) \\ Astronomy Department, University of California, \\ Berkeley, CA 94720, U.S.A. \\ (king@glob.berkeley.edu)
}

\section{Introduction}

It was the feeling of the Scientific Organizing Committee that 50 years after the introduction of the concept of stellar populations it might be desirable to attempt to rationalize their classification and terminology. Toward this end a panel discussion was organized, which was followed by a period of audience participation. Although not all those who contributed to the latter section turned in a written version of their remarks, that section is reported as well as possible here.

\section{Remarks by the Panelists}

\subsection{R. KING:}

After half a century of progress, the subject of stellar populations has developed a richness and a complexity that go far beyond Baade's original concept; as a result present-day discussions are often hampered by the use of a terminology that is outmoded and often both confused and confusing.

I am referring to the terms "Population I" and "Population II." As Baade originally used them, they were a simple dichotomy between two different kinds of HR diagram, that of spiral-arm stars in the Solar neighborhood and that of globular clusters. Interpretation soon followed: the study of accurate color-magnitude arrays of star clusters showed that many differences could be attributed to age, while during the same period spectroscopic studies showed that the globular-cluster stars were deficient in heavy elements. Thus the difference between Populations I and II was identified as a difference in age and in metal abundance. Population II was 
old and metal-poor, compared with the young, metal-rich Population I of the Solar neighborhood. Baade's original two-point dichotomy had thus turned into a sequence-perhaps even a continuous one. The two points had been replaced by a one-dimensional spread, from young, metal-rich to old, metal-poor.

But a single dimension proved to be insufficient. Not only did the metalrich M67 and the metal-poor M3 have main-sequence turnoffs at nearly the same absolute magnitude; the real shock was that the bulge of M31, one of the prototypes of Population II, turned out to be metal-rich, quite unlike the better-known old population in the globular clusters. The distribution of populations was clearly two-dimensional; and of course the age-metallicity plane has since become even better filled in, now that we know that dwarf irregular galaxies have a young, metal-poor population.

Unfortunately history has also left us with another anomaly, the fivepopulation scheme of the 1957 Vatican conference (O'Connell, 1958, pp. 531-533). That conference could hardly have happened at a more unfortunate time. The understanding of stellar populations was going through a major revolution, which might have led to great clarification; but the experts spoke, and they had the misfortune to speak too soon. Their array of populations was one-dimensional, at the very time when it was being realized that the description of stellar populations requires two parameters.

No further meeting of comparable prestige took place afterward, and the Vatican scheme of populations was left in place, inadequate as it was.

Knowledge of populations has increased tremendously since that time, but our language for describing them is in a mess; the question is what to do about it. I believe that one part of the answer is clear: we should recognize that the terms "Population I" and "Population II" no longer have a place in any astronomical discussion except a historical one. Astronomers, unfortunately, still use the terms; but describing a star or a group as "Population II" leaves the listener or reader in complete ambiguity as to whether the meaning is "old" or "low-metallicity". Each of these variables needs to be recognized as independent and treated separately.

(It goes without saying that the Vatican scheme is also of historical interest only.)

The correct choice is to recognize that when we talk about a population we can describe it as old or young and as having high or low metal abundance, and usually in a quantitative way. To say this in a more general way, the question of populations is no longer one of classification but rather of description.

This simple starting point, which I could not recommend more forcefully, is of course not the complete story; there are several complications, which must be dealt with as needed. 
First, "abundance" is clearly not a single quantity; the elements do not vary in lock-step. In many cases one needs to single out some element, or group of elements (e.g., oxygen, the $\alpha$-nuclei, the $s$-process elements, etc.) as being different.

Second, in many populations star formation is a continuing process, and there is a range of ages.

Third, the continuing birth and death of stars can lead to the evolution of abundances with time in a single population; witness the "G-dwarf problem" of the thin-disk population in the Solar neighborhood.

Finally, the population picture is much confused by spatial mixing. The Solar neighborhood is bothered little by this problem, since we have no difficulty distinguishing the interloper stars of the halo population; but in the central bulge of the Galaxy there is severe confusion. It is a dynamical necessity that stars of the halo and the thick disk interpenetrate the disk stars there, and it is not yet clear whether the bulge contains a distinct population of its own rather than merely a mixture of everything that is constrained to pass through that central region.

Notice that I have referred to populations but have refrained from equating them with spatial and kinematic components of the Milky Way. Spatial distributions and kinematic information are indeed very useful in delineating what may indeed be a distinct component, but it is the age and abundance characteristics that describe the population characteristics of that component.

One particular usage of "component" language has led to the worst confusion of all. A number of years ago, some astronomers tried to avoid confusing the stellar halo of the Galaxy with the massive "dark halo" by referring to the stellar component as the "spheroid." But the central bulge of the Galaxy also has a spheroidal shape, and lumping the two under the single term "spheroid" has led to the totally inexcusable implication that the bulge has the same metal-poor population as the halo.

But if there is a single point that I wish to make, it is that populations are not I and II; they are the manifold \{age, abundance\}, and they should be described in the right way, not in the wrong way.

\subsection{S. VAN DEN BERGH:}

Exactly 50 years ago Baade (1944a,b) introduced the concept of Stellar Populations. According to his definitions, Population I consists of stars that have a color-magnitude diagram similar to that of young (metal-rich) open clusters, whereas Population II was defined as having a color-magnitude diagram like that of an old (metal-poor) globular cluster. The first hint of trouble in paradise was provided by Morgan (1956), who showed that the 
dominant population in the nuclear bulge of M31 consisted of old metal-rich stars that exhibited strong CN-bands in their spectra; this showed that not all old stars were metal-poor. During the next decade it became clear that a complete picture of stellar populations could be obtained only by looking at the distribution of stars in the age-abundance plane. Baum (1959) showed that the observed integrated colors of galaxies could be understood in terms of differences in their history of star formation. Most star formation in red ellipticals took place long ago. On the other hand, the rate of star formation in the blue disks of spirals must have been more or less constant during their evolution. Understanding the second coordinate of the age-metallicity plane, i.e., the metallicity distribution of stars, turned out to be more difficult (van den Bergh, 1962). In the Galactic disk metal-rich stars are much more common than would have been expected from a simple "closed-box" evolutionary model. (This was the so-called "G-dwarf problem").

A similar, but less publicized, problem occurs in the halos of M31 and the Galaxy. Stars and clusters with metallicities as high as $[\mathrm{Fe} / \mathrm{H}] \approx-0.8$ occur in the halos of these galaxies, even though only $\sim 2 \%$ of the baryonic mass in these two objects consists of halo stars. The need to produce such high metallicities with so few evolving stars places interesting constraints on theories of halo formation and evolution. By the end of the 1950s it was already clear (Oort, 1958) that a simple two-population model could not account for the diversity exhibited by stellar populations in the Galaxy. In particular Oort stressed the fact that our Milky Way system contains a strong "intermediate Population II," which mediates between the true halo and the old disk. This intermediate Population II has more recently resurfaced in the literature as the "Galactic thick disk" (Gilmore, 1984). Perhaps the strongest early evidence for such an intermediate population is provided by long-period variables. Mira stars with $P<250$ days are associated with (moderately metal-rich) globular clusters, while long-period Miras clearly belong to the old disk population. A puzzling new development has been the discovery (Morrison, 1993) of significant numbers of metal-weak stars with low velocity dispersion at high latitudes. This discovery has been confirmed by Beers and Sommer-Larsen (1994), who find that the low-metallicity tail of the thick disk population extends down to at least $[\mathrm{Fe} / \mathrm{H}] \approx-2.0$. An important constraint on models for the formation of the thick disk (e.g., Quinn, Hernquist and Fullager, 1993) might be provided by observations that give the radial scale-length of the metal-poor thick disk population.

\subsection{K. C. FREEMAN:}

While spiral galaxies appear to have structural components like the old disk, the bulge and the metal-weak halo, we now know that these components 
have a complex history, and I doubt that the traditional population concept provides much useful insight at this time. Here are a few specific examples:

1) One of the most important recent results comes from the paper by Edvardsson et al. (1993) on the age-velocity relation (AVR) and the agemetallicity relation (AMR) for a sample of F stars near the Sun. Their work provides for the first time a sample of stars with internally accurate ages, metallicities and kinematics. For our purpose today, their results on the old disk are particularly interesting. The AVR and AMR for the F stars shows that the younger stars evolve dynamically: their velocity dispersion increases with age up to an age of about $3 \mathrm{Gyr}$. But for stars with ages between about 3 and $10 \mathrm{Gyr}$, there seems to be no further dynamical evolution; it looks as if the dynamical heating has saturated after about $3 \mathrm{Gyr}$ (as one might expect on theoretical grounds). The metallicity distribution of these stars shows a broad distribution with no real change of metallicity with age. From their kinematics and chemical properties, these stars belong to the old disk of the Galaxy. This old disk population is chemically and kinematically very well defined, but includes stars with ages anywhere from 3 to 10 Gyr. It looks as though this "old disk population" has gradually built up over the life of the disk.

2) The role of accretion and mergers in the formation of several components of the Galaxy is not a new concept, although the details are still not entirely clear. Accretion events probably contribute to the formation of the old thick-disk population, the old metal-weak halo and the young intermediate-metallicity halo population recently identified by Preston et al. (1994). Again, it appears that these accretion events are still going on.

3) Disks and bulges appear to be the two main components of disk galaxies, but the distinction between bulges and disks may not be as clear as we have long believed. Recent theoretical work by several groups shows how bulges can form from the inner regions of disks: bars grow, gas is funnelled inwards to form a small central mass which destroys the orbits that define the bar, and the bar rapidly evolves into a more or less axisymmetric bulge. The similarity of the velocity dispersion in the bulge and the inner disk of our Galaxy is consistent with this picture.

4) In our Galaxy, we see several structural stellar components: the disk (old and young), the thick disk, the bulge and the metal-weak halo. But these various components are not all essential ingredients in the formation of disk galaxies. Other spiral galaxies do not show all of these components: some have negligible bulges, some show no evidence for thick disks, and others (like the LMC) do not appear to have a metal-weak halo component.

It still seems useful to identify the various structural components of disk galaxies. Some of these components are direct pointers to factors in the evolution of their parent galaxy; for example, the presence of a disk is 
a clear pointer to dissipational formation. But I think that the population concept is too simple to be really useful at this time.

\subsection{G. GILMORE:}

Currently popular galaxy formation models presume that large galaxies form by accretion of many small units, each of which is a "basic" darkmatter halo, with some amount of baryonic gas entrapped in its potential well. Some of these units may have initiated star formation and chemical evolution before merger, thus looking rather like a gas-rich dwarf galaxy, while some may not. Those essential features of these models which lead to observable consequences are the time at which the last merger of comparable-mass fragments occurred, and the gas fraction of fragments. An expectation is that recent equal-mass mergers generate elliptical galaxies. Thin spiral disks are believed fragile to merging, so that a thick disk, if present, signals the most recent major merger event of a spiral. In all galaxies continued accretion of small units is expected both to provide a continuing source of new material, and to confuse any simple relationships between time, place and chemical abundance evident in stellar populations.

This modern picture supersedes earlier schematic scenarios, while including aspects of both the "ELS monolithic" and "Searle-Zinn lumpy" models that are still discussed in the context of our Galaxy. The blendand-stir process of mergers will have been common at high redshifts, when a rain of dwarf "proto-galaxies" was normal weather for a budding giant. It continues today, at a rate which may still be significant for some galaxies. Examples include the Sagittarius dwarf, which is merging with us harmlessly now, and the Magellanic Clouds, which will merge destructively one day. If this standard picture is valid, most of the mass in the visible parts of the Milky Way arrived as gas, while a significant part of the halo arrived later and/or as stars. The thick disk is then the record of the last substantial Galactic lunch break. What is of interest here is the subsequent chemical and dynamical evolution of this gas, and the dynamical effects, on the stellar distribution formed up to that time, of the arrival of the Galactic building blocks of which the gas was once part. It must be emphasized that alternative models also exist, especially those developed recently by Ferrini et al. (1994) and by Burkert and Hensler (1992), whereby the thick disk is an early pressure-supported phase of thin-disk formation. The real question is whether the Galaxy is like a party balloon, which responds with a structural change to an impulse, or is like a water bed, which absorbs repeated impacts harmlessly.

Stellar populations provide the fossil record of this galactic evolution. Populations can, however, be defined only after analysis, when one knows 
some astrophysics. We observe stars, derive parameters which categorize them, realise that only a relatively small part of parameter space is occupied, and call the occupied parts of possible distribution functions components. Galactic structural components are thus defined more by post hoc convenience than by a priori astrophysics, and need not have a one-to-one correspondence with evolutionary populations. Our aim is see if they do or do not have a simple evolutionary correspondence, with specific focus on the current classification of populations, which includes the old disk, the thick disk, the stellar halo, the central bulge, and the young thin disk. A range of possible evolutionary paths linking some of these "populations / components" is shown in Fig. V of Gilmore (1993).

The important general point for the present discussion is that we are not in a position as yet to define a "stellar population" from first principles as some definable aspect of galactic evolution, and then go to observe it. Rather we must observe, and then proceed deductively. Especial care is required here if current repeated merger/accretion models are valid, as such models are quite inconsistent with many common assumptions in data analysis. The most blatantly violated is the "closed-box" model of chemical evolution. The gas (if any), the stars (if any), and the dark matter (if any) that are present in the accreted object prior to its coalescence in a larger galaxy all go somewhere, quite likely to very different parts of the new, larger galaxy.

Thus, when considering a stellar population, one must consider an evolutionary sequence which allows not only for the history of star formation, chemical enrichment, kinematic modifications, and so on in the parent galaxy, but also for the possibility that there is no single historical path relevant to all stars with apparently similar properties now. Thus, two stars of similar age and kinematics need not have similar chemical evolutionary histories, two stars of similar age and chemical abundance may have very different angular momenta, and so on. Definition of a "stellar population" as a meaningful astrophysical concept then requires knowledge of the history of the star formation rate, the effects and times of chemical mixing in the ISM, the redistribution of angular momentum, the merging history, and so on. Such data are of course never available, nor especially desirable.

Our present aim, in studying stellar populations, is not to label every star with its origin in some multi-dimensional parameter space, subsets of which are called Population 0, Population I, Population II, Population .... Rather, we wish to pose, and to test, broad questions designed to isolate and identify the most important physical processes in galaxy formation and evolution. This is best achieved by defining a "Stellar Population" to be a group of stars which have a predicted set of properties in some subset of multi-dimensional parameter space, designed to isolate some specific astro- 
physical process. One then tests (observationally) the plausibility and/or reality of such an assignment, and thus the importance of the relevant astrophysics.

This process does not follow the letter of the Vatican Conference concept of well-defined "Populations." It does, however, more closely correspond to the reality of astrophysical deduction, and allows for the likely complexity of galactic evolution. Had the globular clusters Omega Centauri and 47 Tucanae happened to pass overhead at Palomar, it might well have been the concept originally favoured!

In any case, the most appropriate summary of the concept of stellar populations, as a valuable though rather blunt tool with which we may increase our understanding of galactic astrophysics, remains that provided by Blaauw in his Introduction to IAU Symposium No. 1: "In the discussions, the terms 'halo,' 'nucleus' and 'disk' are used to indicate different parts of the Galaxy. These general regions are not defined more precisely. Their introduction proved very useful, and one might rather say that their more exact description is one of the problems of galactic research."

\section{General Discussion}

A. Blaauw: There is one particular circumstance of the 1957 Vatican conference that should be noted. As was customary, the semaine d'ètude was specifically instructed to reach conclusions. It was for this reason that the group came out so definitively with its classification scheme.

P. C. van der Kruit: In spite of the diagram of age versus metallicity that seems to fill up completely, we should remember that there is something discrete about populations, namely in flattenings (the two vertical bands in CMD's of deep star counts; component analysis of edge-on spirals).

So, basically there are at least two large-scale components that distinguish themselves in their flattening but have distributions of metallicity and age that may to a large extent overlap. But the basic dichotomy in flattenings is saying something fundamental (van der Kruit and Searle (1982) suggested two discrete major epochs of star formation, but I am now not so sure about it any more) about galaxy formation and evolution.

This is a balance that I would make between recognizing on the one hand the lack of one-to-one relation between age, metallicity and kinematics, and on the other hand the two basic flattenings.

So, although I think we should abolish the terms Populations I and II, I think we can make use of the terms halo and disk, which distinguish themselves by their kinematices and the flattening of their spatial distributions. $J$. R. Mould: A couple of remarks: If we limit ourselves to the two dimensions of age and metallicity, we lose kinematics. Also I noticed that you 
remarked that there was a time when language should be changing rapidly, and I'm not convinced we have passed that time.

I. R. King: I fully appreciate your point. What I was trying to say was that whereas our objective should be to understand the spatial and kinematic components, we should describe their populations in terms of age and metallicity. Kinematics of course offers an opportunity to judge which component a star belongs to.

H. C. Harris: A definition of "open" or "globular" cluster according to luminosity function (suggested earlier by van den Bergh) has the disadvantage that a cluster may change from open to globular as a system evolves through cluster destruction. However, the point is important that the terms "open" and "globular" are no longer well defined. As with other population terms, they should be replaced by more precise terms such as old or young, metal-rich or poor, populous or sparse, and of high or low concentration.

W. E. Harris: Although I will try, as the panelists have recommended to avoid using the terms "Population I and II" again, I would like to mention one other type of stellar population which has not been brought up at this meeting, but which can't be avoided whenever the issues of galaxy formation come up. This other population is always lurking offstage like an uncomfortable ghost, and we usually call it "Population III". It's possible, from the information we now have for example, that many giant $\mathrm{E}$ galaxies may have little or nothing in them that is less than solar metallicity; i.e., the very first generation of stars must have been almost all high-mass objects and thus left no trace behind except for their seeding of the heavy-element abundances in later generations. It's useful to recall that, if one is putting on a production of Shakespeares's Hamlet, the part of the ghost is a very small part, but you can't put on the play without it!

B. M. Poggianti: You have pointed out the necessity to specify both metallicity and age, talking about a stellar population. Shouldn't we specify metallicity in more details? I mean that it looks necessary to me to specify separately iron and $\alpha$-elements. This doesn't look to me a useless complication: both in the Galaxy and much more in ellipticals, this would avoid a lot of confusion that seems to exist. Of course, it is not always possible to specify iron and $\alpha$-elements, both from observations and theory. But let's say which metallicity we are talking about.

I. $R$. King: I agree in principle, but in practice we often lack such detailed information. Also we should avoid an unnecessary deluge of information. To give a metaphoric example, we can refer to a dog as a cocker spaniel without giving its complete pedigree and lineage.

R. Tayler: We all know that there are three factors determining the properties of a star: age, composition, mass. We have heard little about the initial mass function in this Symposium. Is this because variations are thought to 
be unimportant or simply because we do not understand the IMF? In Leon Mestel's absence I must mention magnetic fields. We do not understand the origin of cosmic magnetic fields or their evolution during the histories of galaxies of different types. Star formation is certainly influenced by magnetic fields. The magnetic field might be one factor amongst others which will influence the IMF.

P. Pismis: With all due respect to Baade's proposition (1944a) regarding the existence of two stellar populations in the Galaxy, the proposition was not entirely a new concept. Nearly two decades earlier Bertil Lindblad had given an overall, global picture embracing the distinct groups (subsystems) of stars which compose the Galaxy. Baade's two-population concept may thus be considered to be a corollary to Lindblad's scheme.

In the mid-twenties Strömberg had shown that there existed a well-defined correlation between the size of solar motion determined from different groups of objects and the dispersion of velocities within each group. Lindblad, with admirable insight, suggested a model for the Galaxy which beautifully explained this asymmetry of stellar motions; he proposed that the Galaxy is composed of subsystems, each rotating with a different velocity around a common center, Shapley's Galactic Center.

Baade's proposal of Stellar Populations (1944a, only two at that time) came to strengthen the conjecture of Lindblad. In the course of time one came to know that the kinematic properties as well as the physical properties of the stars in the Galaxy were fairly well correlated with each other.

Despite the epoch-making postulate of Lindblad's subsystem model of the Galaxy this important work has fallen into oblivion. Modern textbooks and treatises make no mention of it, the only exception being Smart's Stellar Dynamics (1938), where the reader can find a brief account of the problem. Even stranger is the fact that Baade in his 1944 paper makes no mention of Lindblad's work, which is obviously akin to his population ideas.

Was Baade unaware of Lindblad's subsystem scheme or did he not want to be aware of it?

N. Samus: For unknown reasons, no one ever mentions a paper by Bottlinger (1933 - especially pp. 34-35), having ideas very much like Baade's (1944a).

I. R. King: I thank the last two speakers for their comments, but I do believe that these remarks are really directed toward the historical section that appears earlier in this volume.

\section{References}

Baade, W., 1944a, Ap. J. 100, 137

Baade, W., 1944b, Ap. J. 100, 147

Baum, W.A., 1959, P. A. S. P. 71, 106

Beers, T.C. and Sommer-Larsen, J., 1994, Ap. J. Suppl., in press 
Bottlinger, K.F., 1933, Veröff. Universitätssternw. Berlin-Babelsberg Bd. X, H. 2

Burkert, A., Truran, J.W. and Hensler, G., 1992, Ap. J. 391, 651

Edvardsson, B., Andersen, J., Lambert, D.L., Nissen, P.E. and Tomkin, J., 1993, A. \& A. 275, 101

Ferrini, F., Molla, M., Pardi, M.C. and Diaz, A.I., 1994, Ap. J. 427, 745

Gilmore, G., 1984, M. N. R. A. S. 207, 223

Gilmore, G., 1992, Minnesota Lectures on the Structure and Dynamics of the Milky Way, ASP Conf. Ser. Vol. 39, ed. R.M. Humphreys, San Francisco: Astron. Soc. of the Pacific, p.55

Morgan, W.W., 1956, P. A. S. P. 68, 509

Morrison, H.L., 1993, A. J. 105, 539

O'Connell, D.J.K. (ed.), 1958, Stellar Populations, Amsterdam: North Holland Publ. Co.

Oort, J.H. 1958, in O'Connell 1958, p.415

Preston, G.W., Beers, T.C. and Shectman, S.A., 1994, A. J. 108, 538

Quinn, P.J., Hernquist, L. and Fullager, D.P., 1993, Ap. J. 403, 74

Smart, W.M., 1938, Stellar Dynamics, Cambridge: Cambridge University Press

van den Bergh, S., 1962, A. J., 67, 486

van der Kruit, P.C. and Searle, L., 1982, A. \& A., 110, 79 